International Journal of Pure and Applied Mathematics

Volume 83 No. 2 2013, 307-329

ISSN: 1311-8080 (printed version); ISSN: 1314-3395 (on-line version)

url: http://www.ijpam.eu

doi: http://dx.doi.org/10.12732/ijpam.v83i2.11

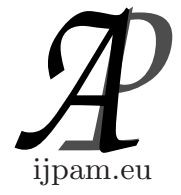

\title{
GENERALIZATION OF $\chi^{2}$ SPACES BY CESÁRO METHOD OF ORDER ONE AND MODULUS FUNCTION
}

\author{
N. Subramanian ${ }^{1 \S}, \mathrm{P}$. Thirunavukkarasu ${ }^{2}, \mathrm{R} . \mathrm{Babu}^{3}$ \\ ${ }^{1}$ Department of Mathematics \\ Sastra University \\ Thanjavur, 613 401, INDIA \\ ${ }^{2}$ P.G. and Research Department of Mathematics \\ Periyar E.V.R. College (Autonomous) \\ Tiruchirappalli, 620 023, INDIA \\ ${ }^{3}$ Department of Mathematics \\ Shanmugha Polytechnic College \\ Thanjavur, 613 401, INDIA
}

\begin{abstract}
In this paper we introduce The Cesàro mean of order one $\chi^{2}$ spaces defined by modulus is associated with a fixed multiplier sequence of non-zero scalars, study their general properties of these spaces and also establish some duals results among them.
\end{abstract}

AMS Subject Classification: 40A05, 40C05, 40D05

Key Words: analytic sequence, modulus function, double sequences, Ces Àro mean, $\chi^{2}$ space

\section{Introduction}

Throughout $w, \chi$ and $\Lambda$ denote the classes of all, gai and analytic scalar valued single sequences, respectively.

We write $w^{2}$ for the set of all complex sequences $\left(x_{m n}\right)$, where $m, n \in \mathbb{N}$,

Received: October 31, 2012

(C) 2013 Academic Publications, Ltd. url: www.acadpubl.eu

${ }^{\S}$ Correspondence author 
the set of positive integers. Then, $w^{2}$ is a linear space under the coordinate wise addition and scalar multiplication.

Some initial works on double sequence spaces is found in Bromwich [4]. Later on, they were investigated by Hardy [5], Moricz [9], Moricz and Rhoades [10], Basarir and Solankan [2], Tripathy [17], Turkmenoglu [19], and many others.

Let us define the following sets of double sequences:

$$
\begin{aligned}
\mathcal{M}_{u}(t) & :=\left\{\left(x_{m n}\right) \in w^{2}: \sup _{m, n \in N}\left|x_{m n}\right|^{t_{m n}}<\infty\right\}, \\
\mathcal{C}_{p}(t) & :=\left\{\left(x_{m n}\right) \in w^{2}: p-\lim _{m, n \rightarrow \infty}\left|x_{m n}-l\right|^{t_{m n}}=1 \text { for some } l \in \mathbb{C}\right\}, \\
\mathcal{C}_{0 p}(t) & :=\left\{\left(x_{m n}\right) \in w^{2}: p-\lim _{m, n \rightarrow \infty}\left|x_{m n}\right|^{t_{m n}}=1\right\}, \\
\mathcal{L}_{u}(t) & :=\left\{\left(x_{m n}\right) \in w^{2}: \sum_{m=1}^{\infty} \sum_{n=1}^{\infty}\left|x_{m n}\right|^{t_{m n}}<\infty\right\}, \\
\mathcal{C}_{b p}(t) & :=\mathcal{C}_{p}(t) \bigcap \mathcal{M}_{u}(t), \\
\mathcal{C}_{0 b p}(t) & =\mathcal{C}_{0 p}(t) \bigcap \mathcal{M}_{u}(t),
\end{aligned}
$$

where $t=\left(t_{m n}\right)$ is the sequence of strictly positive reals $t_{m n}$ for all $m, n \in \mathbb{N}$ and $p-\lim _{m, n \rightarrow \infty}$ denotes the limit in the Pringsheim's sense. In the case $t_{m n}=1$ for all $m, n \in \mathbb{N} ; \mathcal{M}_{u}(t), \mathcal{C}_{p}(t), \mathcal{C}_{0 p}(t), \mathcal{L}_{u}(t), \mathcal{C}_{b p}(t)$ and $\mathcal{C}_{0 b p}(t)$ reduce to the sets $\mathcal{M}_{u}, \mathcal{C}_{p}, \mathcal{C}_{0 p}, \mathcal{L}_{u}, \mathcal{C}_{b p}$ and $\mathcal{C}_{0 b p}$, respectively. Now, we may summarize the knowledge given in some document related to the double sequence spaces. Gökhan and Colak $[21,22]$ have proved that $\mathcal{M}_{u}(t)$ and $\mathcal{C}_{p}(t), \mathcal{C}_{b p}(t)$ are complete paranormed spaces of double sequences and gave the $\alpha-, \beta-, \gamma-$ duals of the spaces $\mathcal{M}_{u}(t)$ and $\mathcal{C}_{b p}(t)$. Quite recently, in her PhD thesis, Zelter [23] has essentially studied both the theory of topological double sequence spaces and the theory of summability of double sequences. Mursaleen and Edely [24] have recently introduced the statistical convergence and Cauchy for double sequences and given the relation between statistical convergent and strongly Cesàro summable double sequences. Nextly, Mursaleen [25] and Mursaleen and Edely [26] have defined the almost strong regularity of matrices for double sequences and applied these matrices to establish a core theorem and introduced the $M$-core for double sequences and determined those four dimensional matrices transforming every bounded double sequences $x=\left(x_{j k}\right)$ into one whose core is a subset of the $M$-core of $x$. More recently, Altay and 
Basar [27] have defined the spaces $\mathcal{B S}, \mathcal{B S}(t), \mathcal{C S} \mathcal{S}_{p}, \mathcal{C} \mathcal{S}_{b p}, \mathcal{C S}$ and $\mathcal{B V}$ of double sequences consisting of all double series whose sequence of partial sums are in the spaces $\mathcal{M}_{u}, \mathcal{M}_{u}(t), \mathcal{C}_{p}, \mathcal{C}_{b p}, \mathcal{C}_{r}$ and $\mathcal{L}_{u}$, respectively, and also examined some properties of those sequence spaces and determined the $\alpha$-duals of the spaces $\mathcal{B S}, \mathcal{B V}, \mathcal{C} \mathcal{S}_{b p}$ and the $\beta(\vartheta)$ - duals of the spaces $\mathcal{C} \mathcal{S}_{b p}$ and $\mathcal{C} \mathcal{S}_{r}$ of double series. Quite recently Basar and Sever [28] have introduced the Banach space $\mathcal{L}_{q}$ of double sequences corresponding to the well-known space $\ell_{q}$ of single sequences and examined some properties of the space $\mathcal{L}_{q}$. Quite recently Subramanian and Misra [29] have studied the space $\chi_{M}^{2}(p, q, u)$ of double sequences and gave some inclusion relations.

Spaces are strongly summable sequences were discussed by Kuttner [31], Maddox [32], and others. The class of sequences which are strongly Cesàro summable with respect to a modulus was introduced by Maddox [8] as an extension of the definition of strongly Cesàro summable sequences. Connor [33] further extended this definition to a definition of strong $A$ - summability with respect to a modulus where $A=\left(a_{n, k}\right)$ is a nonnegative regular matrix and established some connections between strong $A$ - summability, strong $A-$ summability with respect to a modulus, and $A$ - statistical convergence. In [34] the notion of convergence of double sequences was presented by A. Pringsheim. Also, in [35]-[38], and [39] the four dimensional matrix transformation $(A x)_{k, \ell}=$ $\sum_{m=1}^{\infty} \sum_{n=1}^{\infty} a_{k \ell}^{m n} x_{m n}$ was studied extensively by Robison and Hamilton.

We need the following inequality in the sequel of the paper. For $a, b, \geq 0$ and $0<p<1$, we have

$$
(a+b)^{p} \leq a^{p}+b^{p} .
$$

The double series $\sum_{m, n=1}^{\infty} x_{m n}$ is called convergent if and only if the double sequence $\left(s_{m n}\right)$ is convergent, where $s_{m n}=\sum_{i, j=1}^{m, n} x_{i j}(m, n \in \mathbb{N})$ (see[1]).

A sequence $x=\left(x_{m n}\right)$ is said to be double analytic if $\sup _{m n}\left|x_{m n}\right|^{1 / m+n}<$ $\infty$. The vector space of all double analytic sequences will be denoted by $\Lambda^{2}$. A sequence $x=\left(x_{m n}\right)$ is called double gai sequence if $\left((m+n) !\left|x_{m n}\right|\right)^{1 / m+n} \rightarrow 0$ as $m, n \rightarrow \infty$. The double gai sequences will be denoted by $\chi^{2}$. Let $\phi=$ $\{$ allfinitesequences $\}$.

Consider a double sequence $x=\left(x_{i j}\right)$. The $(m, n)^{t h}$ section $x^{[m, n]}$ of the sequence is defined by $x^{[m, n]}=\sum_{i, j=0}^{m, n} x_{i j} \Im_{i j}$ for all $m, n \in \mathbb{N}$, where $\Im_{i j}$ denotes the double sequence whose only non zero term is a $\frac{1}{(i+j) !}$ in the $(i, j)^{t h}$ place for each $i, j \in \mathbb{N}$.

An FK-space(or a metric space) $X$ is said to have AK property if $\left(\Im_{m n}\right)$ is a Schauder basis for $X$. Or equivalently $x^{[m, n]} \rightarrow x$.

An FDK-space is a double sequence space endowed with a complete metriz- 
able; locally convex topology under which the coordinate mappings $x=\left(x_{k}\right) \rightarrow$ $\left(x_{m n}\right)(m, n \in \mathbb{N})$ are also continuous.

Orlicz[13] used the idea of Orlicz function to construct the space $\left(L^{M}\right)$. Lindenstrauss and Tzafriri [7] investigated Orlicz sequence spaces in more detail, and they proved that every Orlicz sequence space $\ell_{M}$ contains a subspace isomorphic to $\ell_{p}(1 \leq p<\infty)$. subsequently, different classes of sequence spaces were defined by Parashar and Choudhary [14], Mursaleen et al. [11], Bektas and Altin [3], Tripathy et al. [18], Rao and Subramanian [15], and many others. The Orlicz sequence spaces are the special cases of Orlicz spaces studied in [6].

Recalling [13] and [6], an Orlicz function is a function $M:[0, \infty) \rightarrow[0, \infty)$ which is continuous, non-decreasing, and convex with $M(0)=0, M(x)>0$, for $x>0$ and $M(x) \rightarrow \infty$ as $x \rightarrow \infty$. If convexity of Orlicz function $M$ is replaced by subadditivity of $M$, then this function is called modulus function, defined by Nakano [12] and further discussed by Ruckle [16] and Maddox [8], and many others.

An modulus function $M$ is said to satisfy the $\Delta_{2}-$ condition for small $u$ or at 0 if for each $k \in \mathbb{N}$, there exist $R_{k}>0$ and $u_{k}>0$ such that $M(k u) \leq R_{k} M(u)$ for all $u \in\left(0, u_{k}\right]$. Moreover, an modulus function $M$ is said to satisfy the $\Delta_{2}-$ condition if and only if

$$
\limsup _{u \rightarrow 0+} \frac{M(2 u)}{M(u)}<\infty
$$

Two Modulus functions $M_{1}$ and $M_{2}$ are said to be equivalent if there are positive constants $\alpha, \beta$ and $b$ such that

$$
M_{1}(\alpha u) \leq M_{2}(u) \leq M_{1}(\beta u) \text { for all } u \in[0, b] .
$$

An modulus function $M$ can always be represented in the following integral form

$$
M(u)=\int_{0}^{u} \eta(t) d t
$$

where $\eta$, the kernel of $M$, is right differentiable for $t \geq 0, \eta(0)=0, \eta(t)>0$ for $t>0, \eta$ is non-decreasing and $\eta(t) \rightarrow \infty$ as $t \rightarrow \infty$ whenever $\frac{M(u)}{u} \uparrow \infty$ as $u \uparrow \infty$.

Consider the kernel $\eta$ associated with the modulus function $M$ and let

$$
\mu(s)=\sup \{t: \eta(t) \leq s\} .
$$

Then $\mu$ possesses the same properties as the function $\eta$. Suppose now

$$
\Phi=\int_{0}^{x} \mu(s) d s .
$$


Then, $\Phi$ is an modulus function. The functions $M$ and $\Phi$ are called mutually complementary Orlicz functions.

Now, we give the following well-known results.

Let $M$ and $\Phi$ are mutually complementary modulus functions. Then, we have:

(i) For all $u, y \geq 0$,

$$
u y \leq M(u)+\Phi(y) \quad \text { (Young's inequality); }
$$

(ii) For all $u \geq 0$,

$$
u \eta(u)=M(u)+\Phi(\eta(u)) ;
$$

(iii) For all $u \geq 0$, and $0<\lambda<1$,

$$
M(\lambda u) \leq \lambda M(u) .
$$

Lindenstrauss and Tzafriri [7] used the idea of Orlicz function to construct Orlicz sequence space

$$
\ell_{M}=\left\{x \in w: \sum_{k=1}^{\infty} M\left(\frac{\left|x_{k}\right|}{\rho}\right)<\infty, \text { for some } \rho>0\right\},
$$

The space $\ell_{M}$ with the norm

$$
\|x\|=\inf \left\{\rho>0: \sum_{k=1}^{\infty} M\left(\frac{\left|x_{k}\right|}{\rho}\right) \leq 1\right\},
$$

becomes a Banach space which is called an Orlicz sequence space. For $M(t)=$ $t^{p}(1 \leq p<\infty)$, the spaces $\ell_{M}$ coincide with the classical sequence space $\ell_{p}$.

If $X$ is a sequence space, we give the following definitions:

(i) $X^{\prime}=$ the continuous dual of $X$;

(ii) $X^{\alpha}=\left\{a=\left(a_{m n}\right): \sum_{m, n=1}^{\infty}\left|a_{m n} x_{m n}\right|<\infty\right.$, for each $\left.x \in X\right\}$;

(iii) $X^{\beta}=\left\{a=\left(a_{m n}\right): \sum_{m, n=1}^{\infty} a_{m n} x_{m n}\right.$ is convegent, for each $\left.x \in X\right\}$;

(iv) $X^{\gamma}=\left\{a=\left(a_{m n}\right): \sup _{m n} \geq 1\left|\sum_{m, n=1}^{M, N} a_{m n} x_{m n}\right|<\infty\right.$, for each $\left.x \in X\right\}$;

(v) Let $X$ be an FK-space $\supset \phi$; then $X^{f}=\left\{f\left(\Im_{m n}\right): f \in X^{\prime}\right\}$;

(vi) $X^{\delta}=\left\{a=\left(a_{m n}\right): \sup _{m n}\left|a_{m n} x_{m n}\right|^{1 / m+n}<\infty\right.$, for each $\left.x \in X\right\}$;

$X^{\alpha} \cdot X^{\beta}, X^{\gamma}$ are called $\alpha-$ (or Köthe-Toeplitz)dual of $X, \beta$-(or generalizedKöthe-Toeplitz) dual of $X, \gamma-$ dual of $X, \delta$-dual of $X$ respectively. $X^{\alpha}$ is defined by Gupta and Kamptan [20]. It is clear that $x^{\alpha} \subset X^{\beta}$ and $X^{\alpha} \subset X^{\gamma}$, 
but $X^{\beta} \subset X^{\gamma}$ does not hold, since the sequence of partial sums of a double convergent series need not to be bounded.

The notion of difference sequence spaces (for single sequences) was introduced by Kizmaz [30] as follows

$$
Z(\Delta)=\left\{x=\left(x_{k}\right) \in w:\left(\Delta x_{k}\right) \in Z\right\}
$$

for $Z=c, c_{0}$ and $\ell_{\infty}$, where $\Delta x_{k}=x_{k}-x_{k+1}$ for all $k \in \mathbb{N}$.

Here $c, c_{0}$ and $\ell_{\infty}$ denote the classes of convergent,null and bounded sclar valued single sequences respectively. The difference space $b v_{p}$ of the classical space $\ell_{p}$ is introduced and studied in the case $1 \leq p \leq \infty$ by BaŞar and Altay in [42] and in the case $0<p<1$ by Altay and BaŞar in [43]. The spaces $c(\Delta), c_{0}(\Delta), \ell_{\infty}(\Delta)$ and $b v_{p}$ are Banach spaces normed by

$$
\|x\|=\left|x_{1}\right|+\sup _{k \geq 1}\left|\Delta x_{k}\right| \text { and }\|x\|_{b v_{p}}=\left(\sum_{k=1}^{\infty}\left|x_{k}\right|^{p}\right)^{1 / p},(1 \leq p<\infty) .
$$

Later on the notion was further investigated by many others. We now introduce the following difference double sequence spaces defined by

$$
Z(\Delta)=\left\{x=\left(x_{m n}\right) \in w^{2}:\left(\Delta x_{m n}\right) \in Z\right\}
$$

where $Z=\Lambda^{2}, \chi^{2}$ and $\Delta x_{m n}=\left(x_{m n}-x_{m n+1}\right)-\left(x_{m+1 n}-x_{m+1 n+1}\right)=x_{m n}-$ $x_{m n+1}-x_{m+1 n}+x_{m+1 n+1}$ for all $m, n \in \mathbb{N}$.

In the present paper, we assume that $\eta=\left(\lambda_{m n}\right)$ is the sequence of non-zero complex numbers. Then for a sequence space $E$, the multiplier sequence space $E(\eta)$ associated with the multiplier sequence $\eta$ is defined by

$$
E(\eta)=\left\{x=\left(x_{m n}\right) \in w^{2}: \eta x=\left(\lambda_{m n} x_{m n}\right) \in E\right\}
$$

The scope for the studies on sequence spaces was extended by using the notion of associated multiplier sequences G. Goes and S. Goes defined the differentiated sequence space $d E$ and integrated sequence space $\int E$ for a given sequence space $E$, using the multiplier sequences $\left(k^{-1}\right)$ and $(k)$ in [47], respectively. A multiplier sequence can be used to accelerate the convergence of the sequences in some spaces.

In some sense, it can be viewed as a catalyst, which is used to accelerate the process of chemical reaction. Sometimes the associated multiplier sequence delays the rate of convergence of a sequence. Thus it also covers a larger class of sequences for study. by

Let $A=\left(a_{k, \ell}^{m n}\right)$ be the Cesàro four dimensional matrix of order one defined

$$
a_{k \ell}^{m n}=\left\{\begin{array}{l}
\frac{1}{(m+1)(n+1)}, 0 \leq k<m \quad \text { and } \quad 0 \leq \ell \leq n, \\
0, k>m, \ell>n .
\end{array}\right.
$$


for all $m, n, k, \ell \in \mathbb{N}$.

\section{Definitions and Preliminaries}

Definition. A modulus function was introduced by Nakano [12]. We recall that a modulus $f$ is a function from $[0, \infty) \rightarrow[0, \infty)$, such that:

(1) $f(x)=0$ if and only if $x=0$;

(2) $f(x+y) \leq f(x)+f(y)$, for all $x \geq 0, y \geq 0$;

(3) $f$ is increasing;

(4) $f$ is continuous from the right at 0 . Since $|f(x)-f(y)| \leq f(|x-y|)$, it follows from here that $f$ is continuous on $[0, \infty)$.

Defintion. Let $A=\left(a_{k, \ell}^{m n}\right)$ denote a four dimensional summability method that maps the complex double sequences $x$ into the double sequence $A x$ where the $k, \ell-$ th term to $A x$ is as follows:

$$
(A x)_{k \ell}=\sum_{m=1}^{\infty} \sum_{n=1}^{\infty} a_{k \ell}^{m n} x_{m n}
$$

such transformation is said to be nonnegative if $a_{\mathrm{k \ell}}^{m n}$ is nonnegative.

The notion of regularity for two dimensional matrix transformations was presented by Silverman [40] and Toeplitz [41]. Following Silverman and Toeplitz, Robison and Hamilton presented the following four dimensional analog of regularity for double sequences in which they both added an adiditional assumption of boundedness. This assumption was made because a double sequence which is $P$ - convergent is not necessarily bounded.

Definition A double sequence $x=\left(x_{m n}\right)$ has a Pringsheim limit $L$ (denoted by $P-\lim x=L)$ provided that given on $\epsilon>0$ there exists $N \in \mathbb{N}$ such that $\left|x_{m n}-L\right|<\epsilon$ whenever $m, n>N$. We shall describe such an $x=\left(x_{m n}\right)$ more briefly as " $P$ - convergent."

Definition Let $f$ be any modulus function and

$$
\delta_{f}(x)=\sum_{i, j} f\left((m+n) !\left|x_{m n}\right|\right)^{1 / m+n},
$$

where $x=\left(x_{m n}\right) \in w^{2}$. Then we define the sets $\tilde{\chi}_{f A}^{2 \eta}$ and $\tilde{\chi}_{f}^{2}$ by 


$$
\begin{aligned}
& \tilde{\chi}_{f A}^{2 \eta}=\left\{x \in w^{2}: \hat{\delta}_{f A}(x)\right. \\
& \left.=\sum_{i, j} f\left(\frac{\left(\sum_{m=0}^{i} \sum_{n=0}^{j} \lambda_{m n}\left((m+n) !\left|x_{m n}\right|\right)^{1 / m+n}\right)}{(i+1)(j+1)}\right) \rightarrow 0 \text { as } m, n \rightarrow \infty\right\}
\end{aligned}
$$

and

$$
\tilde{\chi}_{f}^{2}=\left\{x=\left(x_{m n}\right) \in w^{2}: \delta_{f}(x) \rightarrow 0\right\} .
$$

Definition Let $f$ and $\Phi$ be mutually complementary functions. Then, we define the set $\chi_{f A}^{2 \eta}$ by

$$
\begin{array}{r}
\chi_{f A}^{2 \eta}=\left\{\sum_{i, j} f\left(\frac{\left(\sum_{m=0}^{i} \sum_{n=0}^{j} \lambda_{m n}\left((m+n) !\left|x_{m n}\right|\right)^{1 / m+n}\right)}{(i+1)(j+1)}\right)^{y_{i j} \rightarrow 0}\right. \\
\text { as } \left.m, n \rightarrow \infty \forall y=\left(y_{i j}\right) \in \tilde{\chi}_{\Phi}\right\},
\end{array}
$$

which is called as modulus function associated with the multiplier sequence $\eta=\left(\lambda_{m n}\right)$ and generated by Cesàro four dimensional matrix of order one.

\section{Main Results}

In this section, we emphasize the sequence spaces $\chi_{f A}^{2 \eta}, \tilde{\chi}_{f A}^{2 \eta}, \chi_{f A}^{\prime 2 \eta}$ and $h_{f A}^{2 \eta}$ and give their some algebraic and topological properties.

Proposition For any modulus function $f$, the inclusion $\tilde{\chi}_{f A}^{2 \eta} \subset \chi_{f A}^{2 \eta}$ holds.

Proof. Let $x=\left(x_{m n}\right) \in \tilde{\chi}_{f A}^{2 \eta}$. Then, since

$$
\sum_{i, j} f\left(\frac{\left(\sum_{m=0}^{i} \sum_{n=0}^{j} \lambda_{m n}\left((m+n) !\left|x_{m n}\right|\right)^{1 / m+n}\right)}{(i+1)(j+1)}\right) \rightarrow 0 \text { as } m, n \rightarrow \infty
$$

we have from (1.2) that

$$
\begin{gathered}
\mid \sum_{i, j} f\left(\frac{\left(\sum_{m=0}^{i} \sum_{n=0}^{j} \lambda_{m n}\left((m+n) !\left|x_{m n}\right|\right)^{1 / m+n}\right)}{(i+1)(j+1)} y^{y_{i j}} \mid\right. \\
\leq \sum_{i, j} f\left(\left|\frac{\left(\sum_{m=0}^{i} \sum_{n=0}^{j} \lambda_{m n}\left((m+n) !\left|x_{m n}\right|\right)^{1 / m+n}\right)}{(i+1)(j+1)}\right|\right)
\end{gathered}
$$




$$
+\sum_{i, j} \Phi\left(\left|y_{i j}\right|\right) \rightarrow 0 \text { as } m, n \rightarrow \infty
$$

for every $y=\left(y_{i j}\right) \in \tilde{\chi}_{\Phi}^{2}$. Thus, $x=\left(x_{m n}\right) \in \chi_{f A}^{2 \eta}$.

Proposition For each $x=\left(x_{m n}\right) \in \chi_{f A}^{2 \eta}$,

$$
\begin{aligned}
\sup \left\{\mid \sum_{i, j} f\left(\frac{\left(\sum_{m=0}^{i} \sum_{n=0}^{j} \lambda_{m n}\left((m+n) !\left|x_{m n}\right|\right)^{1 / m+n}\right)}{(i+1)(j+1)}\right)^{y_{i j} \mid:}\right. & \\
& \delta(\Phi, y) \leq 1\}
\end{aligned}
$$

$\rightarrow 0$ as $m, n \rightarrow \infty$.

Proof. Suppose that (3.1) does not hold. Then for each $p, q \in \mathbb{N}$, there exists $y^{p q}$ with $\delta\left(\Phi, y^{p q}\right) \leq 1$ such that

$$
\mid \sum_{i, j} f\left(\frac{\left(\sum_{m=0}^{i} \sum_{n=0}^{j} \lambda_{m n}\left((m+n) !\left|x_{m n}\right|\right)^{1 / m+n}\right)}{(i+1)(j+1)} y_{i j}^{p q} \mid>2^{p+q+2}\right.
$$

With out loss of generality, we can assume that

$$
f\left(\frac{\sum_{m=0}^{i} \sum_{n=0}^{j} \lambda_{m n}\left((m+n) !\left|x_{m n}\right|\right)^{1 / m+n}}{(i+1)(j+1)} y^{p q}\right) \geq 0 .
$$

Now, we can define a sequence $z=\left(z_{i j}\right)$ by $z_{i j}=\sum_{p} \sum_{q} \frac{y_{i j}^{p q}}{2^{p+q+2}}$ for all $i, j \in \mathbb{N}$. By convexity of $\Phi$ we have

$$
\begin{aligned}
\Phi\left(\sum_{p=0}^{r} \sum_{q=0}^{s} \frac{y_{i j}^{p q}}{2^{p+q+2}}\right) & \leq \Phi\left[\frac{1}{2^{2}} y_{i j}^{00}+\frac{1}{2^{4}} y_{i j}^{11}+\cdots+\frac{1}{2^{r+s+2}} y_{i j}^{r s}\right] \\
& \leq \frac{1}{2^{2}}\left[\Phi\left(y_{i j}^{00}\right)+\Phi\left(\frac{y_{i j}^{11}}{2^{2}}+\cdots+\frac{y_{i j}^{r s}}{2^{r+j}}\right)\right] \\
& \leq \cdots \leq \sum_{p=0}^{r} \sum_{q=0}^{s} \frac{1}{2^{p+q+2}} \Phi\left(y_{i j}^{p q}\right),
\end{aligned}
$$

for any positive integer $(r, s)$. Hence, using the continuity of $\Phi$, we have

$$
\delta(\Phi, z)=\sum_{p} \sum_{q} \Phi\left(z_{p q}\right) \leq \sum_{p=0} \sum_{q=0} \frac{1}{2^{p+q+2}} \Phi\left(y_{i j}^{p q}\right) \leq \sum_{p} \sum_{q} \frac{1}{2^{p+q+2}}=\frac{1}{4} .
$$


But for every $r, s \in \mathbb{N}$, it holds

$$
\begin{aligned}
& \left.\sum_{i, j} f\left(\frac{\left(\sum_{m=0}^{i} \sum_{n=0}^{j} \lambda_{m n}\left((m+n) !\left|x_{m n}\right|\right)^{1 / m+n}\right)}{(i+1)(j+1)}\right)^{z_{i j}}\right) \\
& \geq \sum_{i, j} f\left(\frac{\left(\sum_{m=0}^{i} \sum_{n=0}^{j} \lambda_{m n}\left((m+n) !\left|x_{m n}\right|\right)^{1 / m+n}\right)}{(i+1)(j+1)} \sum_{q=0}^{r} \frac{y_{i j}^{p q}}{2^{p+q+2}}\right. \\
& =\sum_{p=0}^{r} \sum_{q=0}^{s} \sum_{i, j} f\left(\frac{\left(\sum_{m=0}^{i} \sum_{n=0}^{j} \lambda_{m n}\left((m+n) !\left|x_{m n}\right|\right)^{1 / m+n}\right)}{(i+1)(j+1)}\right) \frac{y_{i j}^{p q}}{2^{p+q+2}} \\
& \geq r s .
\end{aligned}
$$

Hence $\sum_{m=0}^{i} \sum_{n=0}^{j} f\left(\frac{\lambda_{m n}\left((m+n) !\left|x_{m n}\right|\right)^{1 / m+n}}{(i+1)(j+1)}\right) z_{i j}$ diverges and this implies that $x \notin \chi_{f A}^{2 \eta}$, a contradiction. This completes the proof.

Proposition $\chi_{f A}^{2 \eta}$ is a metric linear under the metric $d(., .)_{f}^{A}$ defined by

$$
\begin{aligned}
d(x, y)_{f}^{A}= & \\
\sup _{m n}\left\{i n f \left(\sum_{i, j} f\left(\frac{\left|\sum_{m=0}^{i} \sum_{n=0}^{j} \lambda_{m n}\left((m+n) !\left|x_{m n}-y_{m n}\right|\right)^{1 / m+n}\right|}{(i+1)(j+1)}\right)\right.\right. & \leq 1\}
\end{aligned}
$$

Proof. Clearly $d(x, y)_{f}^{A}=0$ if $x=y$. Now, suppose that $d(x, y)_{f}^{A}=0$. Then, we have

$$
\begin{array}{r}
\sup _{m n}\left\{\operatorname { i n f } \left(\sum_{i, j} f\left(\frac{\left|\sum_{m=0}^{i} \sum_{n=0}^{j} \lambda_{m n}\left((m+n) !\left|x_{m n}-y_{m n}\right|\right)^{1 / m+n}\right|}{(i+1)(j+1)} \mid\right)\right.\right. \\
\leq 1\}=0,
\end{array}
$$

which implies that

$$
f\left(\frac{\left|\sum_{m=0}^{i} \sum_{n=0}^{j} \lambda_{m n}\left((m+n) !\left|x_{m n}-y_{m n}\right|\right)^{1 / m+n}\right|}{(i+1)(j+1)}\right) \leq 1=0,
$$


for all $i, j \in \mathbb{N}$. It follows that $\lambda_{m n}\left((m+n) !\left|x_{m n}-y_{m n}\right|\right)^{1 / m+n}=0$ for all $i, j \in \mathbb{N}$. Hence $x_{m n}-y_{m n}=0$, since $\left(\lambda_{m n}\right)$ is a sequence of non-zero scalars.

Hence, we have

$$
\begin{aligned}
& d(x, y)_{f}^{A} \\
= & \sup _{m n}\left\{\inf \left(\sum_{i, j} f\left(\frac{\left|\sum_{m=0}^{i} \sum_{n=0}^{j} \lambda_{m n}\left((m+n) !\left|x_{m n}-y_{m n}\right|\right)^{1 / m+n}\right|}{(i+1)(j+1)}\right) \leq 1\right\}\right. \\
\leq & \sup _{m n}\left\{\inf \left(\sum_{i, j} f\left(\frac{\left|\sum_{m=0}^{i} \sum_{n=0}^{j} \lambda_{m n}\left((m+n) !\left|x_{m n}-0\right|\right)^{1 / m+n}\right|}{(i+1)(j+1)}\right) \leq 1\right\}\right. \\
& +\sup _{m n}\left\{\inf \left(\sum_{i, j} f\left(\frac{\left|\sum_{m=0}^{i} \sum_{n=0}^{j} \lambda_{m n}\left((m+n) !\left|y_{m n}-0\right|\right)^{1 / m+n}\right|}{(i+1)(j+1)}\right) \leq 1\right\},\right.
\end{aligned}
$$

which gives that $d(x, y)_{f}^{A} \leq d(x, 0)_{f}^{A}+d(y, 0)_{f}^{A}$.

Finally, let $\alpha$ be any scalar. Then

$$
\begin{aligned}
& d(\alpha(x, y))_{f}^{A} \\
&= \sup _{m n}\left\{\inf \left(\sum_{i, j} f\left(\frac{\left|\sum_{m=0}^{i} \sum_{n=0}^{j} \alpha \lambda_{m n}\left((m+n) !\left|x_{m n}-y_{m n}\right|\right)^{1 / m+n}\right|}{(i+1)(j+1)}\right)\right) \leq 1\right\} \\
&=|\alpha| d((x, y))_{f}^{A} .
\end{aligned}
$$

This completes the proof.

Definition For any modulus function $f$, we define

$$
=\left\{\sum_{i, j} f\left(\frac{\left(\sum_{m=0}^{i} \sum_{n=0}^{j} \lambda_{m n}\left((m+n) !\left|x_{m n}\right|\right)^{1 / m+n}\right)}{(i+1)(j+1)}\right)^{\prime 2 \eta} \rightarrow 0 \text { as } m, n \rightarrow \infty\right\} .
$$

Proposition. The following statements hold:

(i) $\chi_{f A}^{\prime 2 \eta}$ is a metric linear space under the metric $d(., .)_{f}^{A}$ defined by (3.2);

(ii) $\chi_{f A}^{\prime 2 \eta}$ is a Banach metric under the metric defined by (3.2);

(iii) $\chi_{f A}^{\prime 2 \eta}$ is a $B K$ space under the metric defined by (3.2). 
Proof. (i) Since the proof is similar to the proof of Proposition (3.3). Therefore the proof is omitted.

(ii) Let $\left(x^{s}\right)$ be any Cauchy sequence in the space $\chi_{f A}^{\prime 2 \eta}$. Let $\delta>0$ be fixed and $r>0$ be given such that $0<\epsilon<1$ and $r \delta \geq 1$. Then, there exists a positive integer $n_{0}$ such that $d\left(x^{s}, x^{t}\right)_{f}^{A}<\frac{\epsilon}{r \delta}$ for all $s, t \geq n_{0}$. Hence the equation (3.2), we get

$$
\begin{array}{r}
\sup _{m n}\left\{i n f\left(\sum_{i, j} f\left(\frac{\left|\sum_{m=0}^{i} \sum_{n=0}^{j} \lambda_{m n}\left((m+n) !\left|x_{m n}^{s}-x_{m n}^{t}\right|\right)^{1 / m+n}\right|}{(i+1)(j+1)}\right)\right) \leq 1\right\} \\
<\frac{\epsilon}{r \delta},
\end{array}
$$

for all $s, t \geq u_{0} v_{0}$. This implies that

$$
\sum_{i, j} f\left(\frac{\left|\sum_{m=0}^{i} \sum_{n=0}^{j} \lambda_{m n}\left((m+n) !\left|x_{m n}^{s}-x_{m n}^{t}\right|\right)^{1 / m+n}\right|}{d\left(x^{s}, x^{t}\right)_{f}^{A}(i+1)(j+1)} \mid \leq 1,\right.
$$

for all $s, t \geq u_{0} v_{0}$. It follows that

$$
f\left(\frac{\left|\sum_{m=0}^{i} \sum_{n=0}^{j} \lambda_{m n}\left((m+n) !\left|x_{m n}^{s}-x_{m n}^{t}\right|\right)^{1 / m+n}\right|}{d\left(x^{s}, x^{t}\right)_{f}^{A}(i+1)(j+1)} \mid \leq 1,\right.
$$

for all $s, t \geq u_{0} v_{0}$ and for all $i, j \in \mathbb{N}$. For $r>0$ with $f\left(\frac{r \delta}{2}\right) \geq 1$, we have

$$
f\left(\frac{\left|\sum_{m=0}^{i} \sum_{n=0}^{j} \lambda_{m n}\left((m+n) !\left|x_{m n}^{s}-x_{m n}^{t}\right|\right)^{1 / m+n}\right|}{d\left(x^{s}, x^{t}\right)_{f}^{A}(i+1)(j+1)} \mid \leq f\left(\frac{r \delta}{2}\right)\right.
$$

for all $s, t \geq u_{0} v_{0}$ and for all $i, j \in \mathbb{N}$. Since $f$ is non-decreasing, we have

$$
\frac{\left|\sum_{m=0}^{i} \sum_{n=0}^{j} \lambda_{m n}\left((m+n) !\left|x_{m n}^{s}-x_{m n}^{t}\right|\right)^{1 / m+n}\right|}{(i+1)(j+1)} \leq \frac{r \delta}{2} \frac{\epsilon}{r \delta}=\frac{\epsilon}{2},
$$

for all $s, t \geq u_{0} v_{0}$ and for all $i, j \in \mathbb{N}$. Hence, $\left(\lambda_{m n} x_{m n}^{s}\right)$ is a Cauchy sequence in $\mathbb{C}$ for all $i, j \in \mathbb{N}$ which implies that it is a convergent sequence in $\mathbb{C}$ for all $i, j \in \mathbb{N}$.

Let $\lim _{s \rightarrow \infty} \lambda_{m n} x_{m n}^{s}=x_{m n}$ for all $m, n \in \mathbb{N}$. Using the continuity of an modulus function, we have 


$$
\sup _{m n}\left\{\inf \left(\sum_{i, j} f\left(\frac{\left|\sum_{m=0}^{i} \sum_{n=0}^{j} \lambda_{m n}\left((m+n) !\left|x_{m n}^{s}-x_{m n}^{t}\right|\right)^{1 / m+n}\right|}{(i+1)(j+1)}\right)\right)^{\leq 1\}}\right.
$$

for all $s \geq u_{0}$, as $j \rightarrow \infty$. It follows that $\left((m+n) !\left|x_{m n}^{s}-x_{m n}\right|\right)^{1 / m+n} \in \chi_{f A}^{\prime 2 \eta}$. Since $x^{s}$ is in the space $\chi_{f A}^{\prime 2 \eta}$ and $\chi_{f A}^{\prime 2 \eta}$ is a linear space, we have $x=\left(x_{m n}\right) \in \chi_{f A}^{\prime 2 \eta}$.

(iii) From the above proof, one can easily conclude that $d\left(x^{s}, 0\right)_{f}^{A} \rightarrow 0$ as $s \rightarrow \infty$, which implies that $x_{m n}^{s} \rightarrow 0$ as $m, n \rightarrow \infty$ for each $s \in \mathbb{N}$. This completes the proof.

Proposition Let $f$ be an modulus function. Then, $\left(\chi_{f A}^{\prime 2 \eta}, d(., .)_{f}^{A}\right)$ is an $A K-B K$ space.

Proof. Now we show that $\chi_{f A}^{\prime 2 \eta}$ is an AK space. Let $x=\left(x_{m n}\right) \in \chi_{f A}^{\prime 2 \eta}$. We can find $u_{0} v_{0}$ such that

$$
f\left(\left(\frac{\left|\sum_{m=0}^{i} \sum_{n=0}^{j} \lambda_{m n}\left((m+n) !\left|x_{m n}\right|\right)^{1 / m+n}\right|}{(i+1)(j+1)}\right) \leq 1\right) \rightarrow 0 \text { as } m, n \rightarrow \infty .
$$

Define the $(m, n)^{t h}$ section $x^{[m, n]}$ of the sequence is defined by $x^{[m, n]}=$ $\sum_{i, j=0}^{m, n} x_{i j} \Im_{i j}$ for all $m, n \in \mathbb{N}$; where $\Im_{i j}$ denotes the double sequence whose only non zero term is a $\frac{1}{(i+j) !}$ in the $(i, j)^{t h}$ place for each $i, j \in \mathbb{N}$. Hence for $m, n \geq u_{0} v_{0}$, it holds

$$
\begin{aligned}
& d\left(x, x^{[u v]}\right)_{f}^{A} \\
& =\sup _{m n}\left\{\inf \left(\sum_{i, j} f\left(\frac{\left|\sum_{m=0}^{i} \sum_{n=0}^{j} \lambda_{m n}\left((m+n) !\left|x_{m n}\right|\right)^{1 / m+n}\right|}{(i+1)(j+1)}\right)\right) \leq 1:\right. \\
& m \geq u, n \geq v\} \rightarrow 0 \text { as }[u v] \rightarrow \infty .
\end{aligned}
$$

Therefore $x^{[u v]} \rightarrow x \in \chi_{f A}^{\prime 2 \eta}$ as $u v \rightarrow \infty$. Thus $\chi_{f A}^{\prime 2 \eta}$ has AK. This completes the proof. 
Next we show that $\chi_{f A}^{\prime 2 \eta}$ is a BK space. Let $x^{[u v]}$ be a sequence in $\chi_{f A}^{\prime 2 \eta}$ such that $d\left(x, x^{[u v]}\right)_{f}^{A} \rightarrow 0$ as $u v \rightarrow \infty$ where $x=\left(x_{m n}\right) \in \chi_{f A}^{\prime 2 \eta}$, i.e

$$
\sum_{i, j} f\left(\frac{\left|\sum_{m=0}^{i} \sum_{n=0}^{j} \lambda_{m n}\left((m+n) !\left|x_{m n}\right|\right)^{1 / m+n}\right|}{(i+1)(j+1)}\right) \rightarrow 0 \text { as } m, n \rightarrow \infty .
$$

There is $[u v]$ such that $d\left(x, x^{[u v]}\right)_{f}^{A} \leq \frac{1}{2}$. Then, using the convexity of $f$, we have

$$
\begin{aligned}
& \sum_{i, j} f\left(\frac{\left|\sum_{m=0}^{i} \sum_{n=0}^{j} \lambda_{m n}\left((m+n) !\left|x_{m n}\right|\right)^{1 / m+n}\right|}{(i+1)(j+1)} \mid=\sum_{i, j}\right. \\
& f\left(2\left|\sum_{m=0}^{i} \sum_{n=0}^{j} \lambda_{m n}\left((m+n) !\left|x_{m n}^{[u v]}\right|\right)^{1 / m+n}\right|\right. \\
& -2\left|\sum_{m=0}^{i} \sum_{n=0}^{j} \lambda_{m n}\left((m+n) !\left|x_{m n}^{[u v]}\right|\right)^{1 / m+n}\right| \\
& \left.-\left|\sum_{m=0}^{i} \sum_{n=0}^{j} \lambda_{m n}\left((m+n) !\left|x_{m n}\right|\right)^{1 / m+n}\right| / 2(i+1)(j+1)\right) \\
& \leq \frac{1}{2} \sum_{i, j} f\left(\frac{2\left|\sum_{m=0}^{i} \sum_{n=0}^{j} \lambda_{m n}\left((m+n) !\left|x_{m n}^{[u v]}\right|\right)^{1 / m+n}\right|}{(i+1)(j+1)}\right)+ \\
& \frac{1}{2} \sum_{i, j} f\left(\frac{2\left|\sum_{m=0}^{i} \sum_{n=0}^{j} \lambda_{m n}\left((m+n) !\left|x_{m n}^{[u v]}-x_{m n}\right|\right)^{1 / m+n}\right|}{(i+1)(j+1)}\right) \\
& \leq \frac{1}{2} \sum_{i, j} f\left(\frac{2\left|\sum_{m=0}^{i} \sum_{n=0}^{j} \lambda_{m n}\left((m+n) !\left|x_{m n}^{[u v]}\right|\right)^{1 / m+n}\right|}{(i+1)(j+1)}\right)+ \\
& \frac{1}{2} \sum_{i, j} f\left(\frac{2\left|\sum_{m=0}^{i} \sum_{n=0}^{j} \lambda_{m n}\left((m+n) !\left|x_{m n}^{[u v]}-x_{m n}\right|\right)^{1 / m+n}\right|}{d\left(x, x^{[u v]}\right)_{f}^{A}(i+1)(j+1)}\right) \rightarrow 0 \text {, }
\end{aligned}
$$

as $m, n \rightarrow \infty$. 
Hence $x=\left(x_{m n}\right) \in \chi_{f A}^{\prime 2 \eta}$ and consequently $\chi_{f A}^{\prime 2 \eta}$ is a BK space.

Proposition. Let $f$ be an modulus function which satisfies $\Delta_{2}-$ condition. Then $\chi_{A}^{\prime 2 \eta} \subset \chi_{f A}^{\prime 2 \eta}$.

Proof. Let

$$
x \in \chi_{A}^{\prime 2 \eta}
$$

Then $\sum_{i, j}\left(\frac{\left|\sum_{m=0}^{i} \sum_{n=0}^{j} \lambda_{m n}\left((m+n) !\left|x_{m n}\right|\right)^{1 / m+n}\right|}{(i+1)(j+1)}\right) \leq \epsilon$ for sufficiently large $m, n$ and every $\epsilon>0$.

$$
\sum_{i, j} f\left(\frac{\left|\sum_{m=0}^{i} \sum_{n=0}^{j} \lambda_{m n}\left((m+n) !\left|x_{m n}\right|\right)^{1 / m+n}\right|}{(i+1)(j+1)}\right) \leq f(\epsilon) \leq f(2 \epsilon)
$$

(because $f$ is non-decreasing)

$$
\Rightarrow \sum_{i, j} f\left(\frac{\left|\sum_{m=0}^{i} \sum_{n=0}^{j} \lambda_{m n}\left((m+n) !\left|x_{m n}\right|\right)^{1 / m+n}\right|}{(i+1)(j+1)}\right) \leq K f(\epsilon)
$$

by the $\Delta_{2}-$ condition for some $K>0$ )

$$
\leq \epsilon
$$

(by defining $\left.f(\epsilon)<\frac{\epsilon}{K}\right)$.

$$
\sum_{i, j} f\left(\frac{\left|\sum_{m=0}^{i} \sum_{n=0}^{j} \lambda_{m n}\left((m+n) !\left|x_{m n}\right|\right)^{1 / m+n}\right|}{(i+1)(j+1)}\right) \rightarrow 0 \text { as } m, n \rightarrow \infty .
$$

Hence

$$
x \in \chi_{f A}^{\prime 2 \eta}
$$

From (3.3) and (3.4) we get $\chi_{A}^{\prime 2 \eta} \subset \chi_{f A}^{\prime 2 \eta}$. This completes the proof.

Proposition. Let $f_{1}$ and $f_{2}$ be two modulus functions. If $f_{1}$ and $f_{2}$ are equivalent then $\chi_{A}^{\prime 2 \eta}=\chi_{f A}^{\prime 2 \eta}$ and the identity map $I:\left(\chi_{A}^{\prime 2 \eta}, d(., .)_{f_{1}}^{A}\right) \rightarrow$ $\left(\chi_{A}^{\prime 2 \eta}, d(., .)_{f_{1}}^{A}\right)$ is a topological isomorphism.

Proof. Let $\alpha, \beta$ and $b$ be constants from (1.3). Since $f_{1}$ and $f_{2}$ are equivalent, it is obvious that (1.3) holds. Let us take $x=\left(x_{m n}\right) \in \chi_{f A}^{\prime 2 \eta}$. Then,

$$
\sum_{i, j} f_{2}\left(\frac{\left|\sum_{m=0}^{i} \sum_{n=0}^{j} \lambda_{m n}\left((m+n) !\left|x_{m n}\right|\right)^{1 / m+n}\right|}{(i+1)(j+1)}\right) \rightarrow 0 \text { as } m, n \rightarrow \infty .
$$


Hence, for some $[u, v] \geq 1$,

$$
\frac{\left|\sum_{m=0}^{i} \sum_{n=0}^{j} \lambda_{m n}\left((m+n) !\left|x_{m n}\right|\right)^{1 / m+n}\right|}{[u, v](i+1)(j+1)} \leq b
$$

for all $i, j \in \mathbb{N}$. Therefore

$$
\begin{aligned}
& \sum_{i, j} f_{1}\left(\frac{\alpha\left|\sum_{m=0}^{i} \sum_{n=0}^{j} \lambda_{m n}\left((m+n) !\left|x_{m n}\right|\right)^{1 / m+n}\right|}{[u, v](i+1)(j+1)}\right) \\
& \leq \sum_{i, j} f_{2}\left(\frac{\left|\sum_{m=0}^{i} \sum_{n=0}^{j} \lambda_{m n}\left((m+n) !\left|x_{m n}\right|\right)^{1 / m+n}\right|}{(i+1)(j+1)}\right) \rightarrow 0 \text { as } m, n \rightarrow \infty .
\end{aligned}
$$

which shows that the inclusion

$$
\chi_{f_{2} A}^{\prime 2 \eta} \subset \chi_{f_{1} A}^{\prime 2 \eta}
$$

holdss. Similarly we can show that the inclusion

$$
\chi_{f_{1} A}^{\prime 2 \eta} \subset \chi_{f_{2} A}^{\prime 2 \eta}
$$

also holds.

By combining the inclusions (3.5) and (3.6), we conclude that $\chi_{f_{1} A}^{\prime 2 \eta}=\chi_{f_{2} A}^{\prime 2 \eta}$.

$\sum_{i, j}\left(f_{2}\left(\frac{\left|\sum_{m=0}^{i} \sum_{n=0}^{j} \lambda_{m n}\left((m+n) !\left|x_{m n}\right|\right)^{1 / m+n}\right|}{d(x, y)_{f_{2}}^{A}(i+1)(j+1)}\right)^{1}\right) \rightarrow 0$ as $m, n \rightarrow \infty$.

we can find $\mu>1$ with $\frac{b}{2} \mu p_{2}\left(\frac{b}{2}\right) \geq 1$, where $p_{2}$ is the kernel associated with $f_{2}$. Hence,

$$
f_{2}\left(\frac{\left|\sum_{m=0}^{i} \sum_{n=0}^{j} \lambda_{m n}\left((m+n) !\left|x_{m n}\right|\right)^{1 / m+n}\right|}{d(x, y)_{f_{2}}^{A}(i+1)(j+1)} \mid \leq \frac{b}{2} \mu p_{2}\left(\frac{b}{2}\right)\right.
$$

for all $i, j \in \mathbb{N}$.

This implies that

$$
\left(\frac{\left|\sum_{m=0}^{i} \sum_{n=0}^{j} \lambda_{m n}\left((m+n) !\left|x_{m n}\right|\right)^{1 / m+n}\right|}{\mu d(x, y)_{f_{2}}^{A}(i+1)(j+1)} \mid \leq b\right.
$$


for all $i, j \in \mathbb{N}$.

Therefore,

$$
\sum_{i, j}\left(f_{1}\left(\frac{\alpha\left|\sum_{m=0}^{i} \sum_{n=0}^{j} \lambda_{m n}\left((m+n) !\left|x_{m n}\right|\right)^{1 / m+n}\right|}{\mu d(x, y)_{f_{2}}^{A}(i+1)(j+1)}\right)^{\leq 1} \rightarrow 0\right.
$$

as $m, n \rightarrow \infty$.

Hence, $d(x, y)_{f_{1}}^{A} \leq\left(\frac{\mu}{\alpha}\right) d(x, y)_{f_{2}}^{A}$. Similarly, we can show that $d(x, y)_{f_{2}}^{A} \leq$ $\beta \gamma d(x, y)_{f_{1}}^{A}$ by choosing $\gamma$ with $\gamma \beta>1$ such that $\gamma \beta\left(\frac{b}{2}\right) p_{1}\left(\frac{b}{2}\right) \geq 1$. Thus $\alpha \mu^{-1} d(x, y)_{f_{1}}^{A} \leq d(x, y)_{f_{2}}^{A} \leq \beta \gamma d(x, y)_{f_{1}}^{A}$. Hence $I$ is topological isomorphism.

Proposition. $\left[\Lambda_{f A}^{\prime 2 \eta}\right]^{\beta}=\left[\Lambda_{f A}^{\prime 2 \eta}\right]^{\alpha}=\left[\Lambda_{f A}^{\prime 2 \eta}\right]^{\gamma}=\eta_{f A}^{\prime 2 \eta}$, where

$$
=\bigcap_{N \in N-\{1\}}\left\{x=\left(x_{m n}\right): \sum_{i, j} \eta_{f A}^{\prime 2 \eta}\left(f\left(\frac{\left.\left|\sum_{m=0}^{i} \sum_{n=0}^{j} \lambda_{m n}\right| x_{m n}\right|^{1 / m+n} \mid}{(i+1)(j+1)}\right)\right)<\infty\right\} .
$$

Proof. (1) First we show that $\eta_{f A}^{\prime 2 \eta} \subset\left[\Lambda_{f A}^{\prime 2 \eta}\right]^{\beta}$. Let $x \in \eta_{f A}^{\prime 2 \eta}$ and $y \in$ $\left[\begin{array}{c}\prime 2 \eta \\ f A\end{array}\right]$. Then we can find a positive integer $N$ such that $\left(\left|y_{m n}\right|^{1 / m+n}\right)<$ $\max \left(1, \sup _{m, n \geq 1}\left|y_{m n}\right|^{1 / m+n}<N\right)$, for all $m, n$. Hence we may write

$$
\begin{aligned}
&\left|\sum \sum x_{m n} y_{m n}\right| \leq \sum \sum\left|x_{m n} y_{m n}\right| \\
& \leq \sum_{i, j}\left(f\left(\frac{\left|\sum_{m=0}^{i} \sum_{n=0}^{j} \lambda_{m n}\right| x_{m n} y_{m n}||}{(i+1)(j+1)}\right)\right) \\
& \leq \sum_{i, j}\left(f\left(\frac{\left|\sum_{m=0}^{i} \sum_{n=0}^{j} \lambda_{m n}\right| x_{m n}\left|N^{m+n}\right|}{(i+1)(j+1)}\right)\right)
\end{aligned}
$$

Since $x \in \eta_{f A}^{\prime 2 \eta}$, the series on the right side of the above inequality is convergent, whence $x \in\left[\Lambda_{f A}^{\prime 2 \eta}\right]^{\beta}$. Hence $\eta_{f A}^{\prime 2 \eta} \subset\left[\Lambda_{f A}^{\prime 2 \eta}\right]^{\beta}$.

Now we show that $\left[\Lambda_{f A}^{\prime 2 \eta}\right]^{\beta} \subset \eta_{f A}^{\prime 2 \eta}$. 
For this, let $x \in\left[\Lambda_{f A}^{\prime 2 \eta}\right]^{\beta}$, and suppose that $x \notin\left[\Lambda_{f A}^{\prime 2 \eta}\right]^{\beta}$. Then there exists a positive integer $N>1$ such that $\sum_{i, j}\left(f\left(\frac{\left|\sum_{m=0}^{i} \sum_{n=0}^{j} \lambda_{m n}\right| x_{m n}\left|N^{m+n}\right|}{(i+1)(j+1)}\right)\right)=\infty$.

If we define $y_{m n}=N^{m+n} \operatorname{Sgn}_{m n}, m, n=1,2,3, \cdots$, then $y \in\left[\Lambda_{f A}^{\prime 2 \eta}\right]^{\beta}$.

But, since

$$
\begin{aligned}
\left|\sum \sum x_{m n} y_{m n}\right|= & \sum \sum\left(f\left(\left|x_{m n} y_{m n}\right|\right)\right) \\
& =\sum_{i, j}\left(f\left(\frac{\left|\sum_{m=0}^{i} \sum_{n=0}^{j} \lambda_{m n}\right| x_{m n}\left|N^{m+n}\right|}{(i+1)(j+1)}\right)\right)=\infty,
\end{aligned}
$$

we get $x \notin\left[\Lambda_{f A}^{\prime 2 \eta}\right]^{\beta}$, which contradicts to the assumption $x \in\left[\Lambda_{f A}^{\prime 2 \eta}\right]^{\beta}$. Therefore $x \in \eta_{f A}^{\prime 2 \eta}$. Therefore $\left[\Lambda_{f A}^{\prime 2 \eta}\right]^{\beta}=\eta_{f A}^{\prime 2 \eta}$.

(ii) and (iii) can be shown in a similar way of (i). Therefore we omit it.

Proposition $\chi_{f A}^{\prime 2 \eta} \subset \Gamma_{f A}^{\prime 2 \eta}$.

Proof. Let $x \in \chi_{f A}^{\prime 2 \eta}$.

Then we have

$$
\left\{\sum_{i, j} f\left(\frac{\left(\sum_{m=0}^{i} \sum_{n=0}^{j} \lambda_{m n}\left((m+n) !\left|x_{m n}\right|\right)^{1 / m+n}\right)}{(i+1)(j+1)}\right) \rightarrow 0 \text { as } m, n \rightarrow \infty\right\} \text {. }
$$

Here, we get

$$
\left\{\sum_{i, j} f\left(\frac{\left(\sum_{m=0}^{i} \sum_{n=0}^{j} \lambda_{m n}\left(\left|x_{m n}\right|\right)^{1 / m+n}\right)}{(i+1)(j+1)} \mid \rightarrow \text { as } m, n \rightarrow \infty\right\} .\right.
$$

Thus we have $x \in \Gamma_{f A}^{\prime 2 \eta}$ and so $\chi_{f A}^{\prime 2 \eta} \subset \Gamma_{f A}^{\prime 2 \eta}$.

Proposition. The $\beta-$ dual space of $\chi_{f A}^{\prime 2 \eta}$ is $\Lambda_{f A}^{\prime 2 \eta}$.

Proof. First, we observe that $\chi_{f A}^{\prime 2 \eta} \subset \Gamma_{f A}^{\prime 2 \eta}$ by Proposition 3.10. Theorefore $\left(\Gamma_{f A}^{\prime 2 \eta}\right)^{\beta} \subset\left(\chi_{f A}^{\prime 2 \eta}\right)^{\beta}$. But $\left(\Gamma_{f A}^{\prime 2 \eta}\right)^{\beta} \stackrel{\subset}{\neq} \Lambda_{f A}^{\prime 2 \eta}$. Hence

$$
\Lambda_{f A}^{\prime 2 \eta} \subset\left(\chi_{f A}^{\prime 2 \eta}\right)^{\beta}
$$


Next we show that $\left(\chi_{f A}^{\prime 2 \eta}\right)^{\beta} \subset \Lambda_{f A}^{\prime 2 \eta}$. Let $y=\left(y_{m n}\right) \in\left(\chi_{f A}^{\prime 2 \eta}\right)^{\beta}$. Consider $f(x)=\sum_{m=1}^{\infty} \sum_{n=1}^{\infty} x_{m n} y_{m n}$ with $x=\left(x_{m n}\right) \in \chi_{f A}^{\prime 2 \eta}$ :

$$
\begin{aligned}
& x=\left[\left(\Im_{m n}-\Im_{m n+1}\right)-\left(\Im_{m+1 n}-\Im_{m+1 n+1}\right)\right] \\
& =\left(\begin{array}{ccccccc}
0 & 0 & \cdots & 0 & 0 & \cdots & 0 \\
0 & 0 & \cdots & 0 & 0 & \cdots & 0 \\
\vdots & & & & & \\
0 & 0 & \cdots & \frac{(i+1)(j+1)}{\lambda_{m n}(m+n) !} & \frac{-(i+1)(j+1)}{\lambda_{m n}(m+n) !} & \cdots & 0 \\
0 & 0 & \cdots & 0 & 0 & \cdots & 0
\end{array}\right) \\
& -\left(\begin{array}{ccccccc}
0 & 0 & \cdots & 0 & 0 & \cdots & 0 \\
0 & 0 & \cdots & 0 & 0 & \cdots & 0 \\
\vdots & & & & & \\
0 & 0 & \cdots & \frac{(i+1)(j+1)}{\lambda_{m n}(m+n) !} & \frac{-(i+1)(j+1)}{\lambda_{m n}(m+n) !} & \cdots & 0 \\
0 & 0 & \cdots & 0 & 0 & \cdots & 0
\end{array}\right),
\end{aligned}
$$

$\left(\frac{\lambda_{m n}\left((m+n) !\left|x_{m n}\right|\right)^{1 / m+n}}{(i+1)(j+1)}\right)$

$$
=\left(\begin{array}{ccccccc}
0 & 0 & \cdots & 0 & 0 & \cdots & 0 \\
0 & 0 & \cdots & 0 & 0 & \cdots & 0 \\
\cdot & & & & & \\
\cdot & & & & & & \\
0 & 0 & \cdots & \frac{(i+1)(j+1)}{\lambda_{m n}(m+n) !} & \frac{-(i+1)(j+1)}{\lambda_{m n}(m+n) !} & \cdots & 0 \\
0 & 0 & \cdots & \frac{(i+1)(j+1)}{\lambda_{m n}(m+n) !} & \frac{(i+1)(j+1)}{\lambda_{m n}(m+n) !} & \cdots & 0 \\
0 & 0 & \cdots & 0 & 0, & \cdots & 0
\end{array}\right) .
$$

Hence converges to zero.

Therefore

$$
\left[\left(\Im_{m n}-\Im_{m n+1}\right)-\left(\Im_{m+1 n}-\Im_{m+1 n+1}\right)\right] \in\left(\begin{array}{c}
\prime 2 \eta \\
\chi_{f A}
\end{array}\right) .
$$

Hence,

$$
d\left(\left(\Im_{m n}-\Im_{m n+1}\right)-\left(\Im_{m+1 n}-\Im_{m+1 n+1}\right), 0\right)=1 .
$$


But

$$
\left|y_{m n}\right| \leq\|f\| d\left(\left(\Im_{m n}-\Im_{m n+1}\right)-\left(\Im_{m+1 n}-\Im_{m+1 n+1}\right), 0\right) \leq\|f\| 1<\infty,
$$

for each $m, n$. Thus $\left(y_{m n}\right)$ is a double Cesàro mean of order one bounded sequence and hence an Cesàro mean of order one analytic sequence. In other words $y \in\left(\Lambda_{f A}^{\prime 2 \eta}\right)$. But $y=\left(y_{m n}\right)$ is arbitrary in $\left(\chi_{f A}^{\prime 2 \eta}\right)^{\beta}$. Therefore

$$
\left(\chi_{f A}^{\prime 2 \eta}\right)^{\beta} \subset\left(\Lambda_{f A}^{\prime 2 \eta}\right)
$$

From (3.7) and (3.8) we get $\left(\chi_{f A}^{\prime 2 \eta}\right)^{\beta}=\left(\Lambda_{f A}^{\prime 2 \eta}\right)$.

\section{References}

[1] T. Apostol, Mathematical Analysis, Addison-wesley, London (1978).

[2] M. Basarir, O. Solancan, On some double sequence spaces, J. Indian Acad. Math., 21, No. 2 (1999), 193-200.

[3] C. Bektas, Y. Altin, The sequence space $\ell_{M}(p, q, s)$ on seminormed spaces, Indian J. Pure Appl. Math., 34, No. 4 (2003), 529-534.

[4] T.J.I'A. Bromwich, An introduction to the theory of infinite series, Macmillan and Co. Ltd., New York (1965).

[5] G.H. Hardy, On the convergence of certain multiple series, Proc. Camb. Phil. Soc., 19 (1917), 86-95.

[6] M.A. Krasnoselskii, Y.B. Rutickii, Convex Functions and Orlicz Spaces, Gorningen, Netherlands (1961).

[7] J. Lindenstrauss, L. Tzafriri, On Orlicz sequence spaces, Israel J. Math., 10 (1971), 379-390.

[8] I.J. Maddox, Sequence spaces defined by a modulus, Math. Proc. Cambridge Philos. Soc, 100, No. 1 (1986), 161-166.

[9] F. Moricz, Extentions of the spaces $c$ and $c_{0}$ from single to double sequences, Acta. Math. Hung., 57, No-s: 1-2 (1991), 129-136. 
[10] F. Moricz, B.E. Rhoades, Almost convergence of double sequences and strong regularity of summability matrices, Math. Proc. Camb. Phil. Soc., 104 (1988), 283-294.

[11] M. Mursaleen, M.A. Khan, Qamaruddin, Difference sequence spaces defined by Orlicz functions, Demonstratio Math., XXXII (1999), 145-150.

[12] H. Nakano, Concave modulars, J. Math. Soc. Japan, 5 (1953), 29-49.

[13] W. Orlicz, Über Raume $\left(L^{M}\right)$, Bull. Int. Acad. Polon. Sci. A (1936), 93107.

[14] S.D. Parashar, B. Choudhary, Sequence spaces defined by Orlicz functions, Indian J. Pure Appl. Math., 25, No. 4 (1994), 419-428.

[15] K. Chandrasekhara Rao, N. Subramanian, The Orlicz space of entire sequences, Int. J. Math. Math. Sci., 68 (2004), 3755-3764.

[16] W.H. Ruckle, FK spaces in which the sequence of coordinate vectors is bounded, Canad. J. Math., 25 (1973), 973-978.

[17] B.C. Tripathy, On statistically convergent double sequences, Tamkang J. Math., 34, No. 3 (2003), 231-237.

[18] B.C. Tripathy, M. Et, Y. Altin, Generalized difference sequence spaces defined by Orlicz function in a locally convex space, J. Anal. Appl., 1, No. 3 (2003), 175-192.

[19] A. Turkmenoglu, Matrix transformation between some classes of double sequences, J. Inst. Math. Comp. Sci. Math. Ser., 12, No. 1 (1999), 23-31.

[20] P.K. Kamthan, M. Gupta, Sequence Spaces and Series, Lecture Notes, Pure and Applied Mathematics, 65, Marcel Dekker, In c., New York (1981).

[21] A. Gökhan, R. Çolak, The double sequence spaces $c_{2}^{P}(p)$ and $c_{2}^{P B}(p), A p p l$. Math. Comput., 157, No. 2 (2004), 491-501.

[22] A. Gökhan, R. Çolak, Double sequence spaces $\ell_{2}^{\infty}$, ibid., 160, No. 1 (2005), 147-153.

[23] M. Zeltser, Investigation of Double Sequence Spaces by Soft and Hard Analitical Methods, Dissertationes Mathematicae Universitatis Tartuensis 25, Tartu University Press, Univ. of Tartu, Faculty of Mathematics and Computer Science, Tartu (2001). 
[24] M. Mursaleen, O.H.H. Edely, Statistical convergence of double sequences, J. Math. Anal. Appl., 288, No. 1 (2003), 223-231.

[25] M. Mursaleen, Almost strongly regular matrices and a core theorem for double sequences, J. Math. Anal. Appl., 293, No. 2 (2004), 523-531.

[26] M. Mursaleen, O.H.H. Edely, Almost convergence and a core theorem for double sequences, J. Math. Anal. Appl., 293, No. 2 (2004), 532-540.

[27] B. Altay, F. BaŞar, Some new spaces of double sequences, J. Math. Anal. Appl., 309, No. 1 (2005), 70-90.

[28] F. BaŞar, Y. Sever, The space $\mathcal{L}_{p}$ of double sequences, Math. J. Okayama Univ, 51 (2009), 149-157.

[29] N. Subramanian, U.K. Misra, The semi normed space defined by a double gai sequence of modulus function, Fasciculi Math., 46 (2010).

[30] H. Kizmaz, On certain sequence spaces, Cand. Math. Bull., 24, No. 2 (1981), 169-176.

[31] B. Kuttner, Note on strong summability, J. London Math. Soc., 21 (1946), 118-122.

[32] I.J. Maddox, On strong almost convergence, Math. Proc. Cambridge Philos. Soc., 85, No. 2 (1979), 345-350.

[33] J. Cannor, On strong matrix summability with respect to a modulus and statistical convergence, Canad. Math. Bull., 32, No. 2 (1989), 194-198.

[34] A. Pringsheim, Zurtheorie derzweifach unendlichen zahlenfolgen, Math. Ann., 53 (1900), 289-321.

[35] H.J. Hamilton, Transformations of multiple sequences, Duke Math. J., 2 (1936), 29-60.

[36] H.J. Hamilton, A Generalization of multiple sequences transformation, Duke Math. J., 4 (1938), 343-358.

[37] H.J. Hamilton, Change of Dimension in sequence transformation, Duke Math. J., 4 (1938), 341-342.

[38] H.J. Hamilton, Preservation of partial Limits in Multiple sequence transformations, Duke Math. J., 4 (1939), 293-297. 
[39] , G.M. Robison, Divergent double sequences and series, Amer. Math. Soc. Trans., 28 (1926), 50-73.

[40] L.L. Silverman, On the Definition of the Sum of a Divergent Series, Unpublished Thesis, University of Missouri Studies, Mathematics Series.

[41] O. Toeplitz, Über allgenmeine linear mittel bridungen, Prace Matemalyczno Fizyczne, Warsaw, 22 (1911).

[42] F. BaŞar, B. Atlay, On the space of sequences of $p$ - bounded variation and related matrix mappings, Ukrainian Math. J., 55, No. 1 (2003), 136-147.

[43] B. Altay, F. BaŞar, The fine spectrum and the matrix domain of the difference operator $\Delta$ on the sequence space $\ell_{p},(0<p<1)$, Commun. Math. Anal., 2, No. 2 (2007), 1-11.

[44] R. Çolak, M. Et, E. Malkowsky, Some Topics of Sequence Spaces, Lecture Notes in Mathematics, Firat Univ. Elazig, Turkey (2004), 1-63; Firat Univ. Press (2004), ISBN: 975-394-0386-6.

[45] H. Dutta, F. BaŞar, A Generalization of Orlicz sequence spaces by cesàro mean of order one, Acta Math. Univ. Commenianae, LXXX, No. 2 (2011), $185-200$.

[46] Ayhan Esi, On some new difference double sequence spaces via Orlicz function, Journal of Advanced Studies in Topology, 2, No. 2 (2011), 16-25.

[47] G. Goes, S. Goes, Sequences of bounded variation and sequences of Fourier coefficients, Math. Z., 118 (1970), 93-102. 
